\title{
III. - ERRATA
}

\section{Errata au Bulletin géodésique}

Bulletin géodésique $n^{\mathrm{n}} 12$.

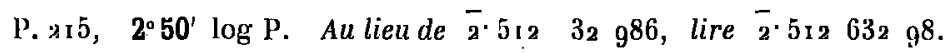

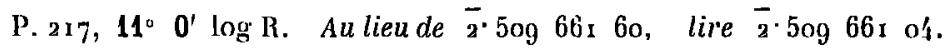
P. $218,20^{\prime \prime} \mathbf{0}^{\prime} \log \mathbf{R}$. Aulieu de $\overline{2} \cdot 5095473_{7}$, lire $\overline{2} \cdot 509543 \quad 37$. P. $226,58^{\circ} 0^{\prime} \log \mathrm{R}$. Au lieu de $\overline{\mathbf{2}} \cdot 508$ 661 80, lire $\overline{\mathbf{2}} \cdot 50866 \mathrm{r}{ }^{8}$. Pour les différences correspondantes, au lieu de 38 a et 385, lire 384 et 383 .

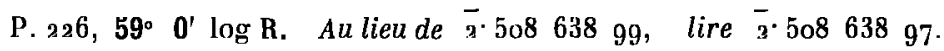
Pour les différences correspondantes, au lieu de 375 ct 378 , lire 377 et 376 . P. 23 2. $88^{\circ} 40^{\prime} \log \mathrm{R}$. Au lieu de la différence 9, lire la différence i 9. P. 248-249. Tableau : Exemple de calcul par des formules dérivćes de celles de Andrae :

Première colonne. Au lieu de $\varepsilon=-4^{\prime \prime}, 144$, lire $\varepsilon=-4^{\prime \prime}, 154$.

Deuxième colonne. Au lieu de $\log \cos \left(z-\frac{2}{3}\right)$, lire $\log \cos \left(z-\frac{2 E}{3}\right)$. P. 248-249. Tableau : Exemple de calcul par les formules de Benoit, etc. Deuxième colonne. Aulieu de $\mathrm{L}=-\mathrm{I} 6^{\circ} 39^{\prime} 45^{\prime \prime}, 49^{2}$, lire $\mathrm{L}=\mathrm{I} 6^{\circ} 39^{\prime \prime} 45^{\prime \prime}, 49^{2}$.

\section{Bulletin géodésique $n^{0} 14$.}

P. 97, ligne 2. Supprimer $\mathbf{1}^{\circ}$.

Bulletin géodesique $n^{\circ} 17$.

P. 3, ligne 22. Après Survey ", ajouler et de l" "American Geophysical Union ".

P. 9, ligne ı . Après Pologne, ajouter el du Service géographique de J'Arméc. 
P. 78. Supprimer les lignes 8 et 9 et les remplacer par :

Au lieu de : " dépasse $"$,

Lirc : " ne dépasse pas".

Au lieu de " ou dans laquelle une (ou plusieurs) de ces valeurs atteint $3^{\prime \prime}$ ou $9 ", 3 n$,

Lire: " ou dans lesquelles très pou de ces valeurs atteignent $3^{\prime \prime}$ ou $9 ", 3$ ".

Dès lors, le texte exact à introduire à la place des sept premières lignes de la page 67 est le suivant :

a des méthodes donnant une grande précision. Comme le critérium le plus immédiat de la valeur d'une triangulation consiste dans les erreurs de fermeture des triangles, nous admettons que le nom de triangulation de premier ordre ne peut s'appliquer qu'aux triangulations dans lesquelles la moyenne des valeurs absolues des crreurs de fermeture ne dépasse pas $3^{\prime \prime}$ ou $9^{\prime \prime}, 3$, ou dans lesquelles très peu de ces valeurs atteignent $3^{\prime \prime}$ ou $.9^{\prime \prime}, 3$." "

P. 109. Lors de l'Assemblée générale de Prague en 1927, la Section de Géodésie de l'Union gćodésique et géophysique internationalc a établi, pour les Nivellements de haute Précision, des règles générales dont elle a recommandé l'adoption aux états adhérents à l'Union. La Commission chargće de cette étude (Commission $\mathrm{n}^{\circ}{ }_{9}$, de Réglementation internationale des Travaux géodésiques) avait pris pour base de son travail des propositions élaborées par M. Ch. Lallemand, Rapporteur général pour les Nivellements de haute Précision (Bulletin géodésique $\mathrm{n}^{\circ} \mathrm{1} 7$, janvier-février-mars Iga8). $^{2}$.

En ce qui concerne le contrôle des opérations au fur et à mesure des calculs, les propositions de M. Lallemand prévoyaient une comparaison méthodique, suivant des règles déterminées, entre les différences de niveau obtenues, à l'aller et au retour, “ de repère à repère, par tronçons de moins d'un kilomètre, et mẻme, si possible, de piquet à piquet " (Ibid., p. 84).

Le Président de la Commission, M. W. Bowie, et divers membres observèrent que les formules de comparaison données par M. Lallemand concernaient, pour la plupart, les discordances par nivelée, alors que plusieurs pays, n'employant pas les mémes piquets au retour qu'à l'aller. ne pouvaient faire porter la comparaison que sur des tronçons beaucoup plus ćtendus (p. Io3).

Je fus donc chargé de préparer des formules applicables aux discordances calculćes aussi bien entre repères qu'entre piquets consécutifs, et équivalentes, dans co dernier cas, à celles de M. Lallemand. Le texte remanié par 
moi fut approuvé par la Commission, puis adopté par la Section de Géodésie (p. rog).

Or, dans ma rédaction, il s'est glissé une légère erreur de plume, par suite d'une confusion entre les mots nivelée rt inlervalle. Cetle erreur se trouverait redressćc par les modifications suivantes, à apporter à la page $\log$ du Bulletin géodésique $\mathrm{n}^{\circ}{ }^{17}$ :

Ligne 4, en remontant. Au lieu de " pour so à 20 intervalles ", lire "pour un à trois kilomètres".

Ligne 3, en remontant. Au lieu de "pour plusieurs centaines d'intervalles », lire " pour plusieurs dizaines de kilomètres $\Perp$.

Lignes $2 \mathrm{ct}$ I, en remontant. Au lieu de « pour un nombre d'intervalles compris entre so et plusicurs centaines ", lire " pour une longueur comprise entre trois et quelques dizaines de kilomètres".

Cette rectification d'une simple crreur matérielle parait. aller de soi. Pour s'en convaincre, il suffit de comparer aux propositions primitives de M. Lallemand (p. 84) le texte élaboré par moi (p. Iog) et de remarquer que j'avais íté chargé, non de modifier lo sens de ces propositions. mais de les rendre applicables à des intervalles différents de la nivelée, et de longucur arbitrairc.

Au reste, si l'un des états adhérents à l'Union voyait à la modification précédente uu inconvénient quelconque, il lui suffirait de soumettre la question à la procbaine Assemblée générale de Stockholm.

$$
\text { Paris, le to juin rgag. Signé : Jean Vignal. }
$$

\section{Bulletin géodésique $n^{\circ} 18$.}

P. 246, ligne 1 . Au lieu de "limités pour" ", lire "limitós par ». P. 419, ligne dernièrc. Au lieu de "4x", lire " 4 เo".

\section{Errata aux tables à 8 décimales des valeurs naturelles des sinus, cosinus et tangentes dans le système décimal, etc.}

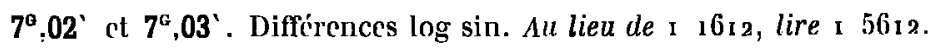
$7^{\mathrm{G}}, 06^{\circ}$ et $7^{\circ}, 07^{\prime}$. Différences $\log$ cos. Au lieu de ${ }_{1} 339$, lire $\mathrm{I} 7^{3} \mathrm{~g}$.

$14^{\circ}, 65^{\prime}$ Log tang. Au lieu de $0.234267_{10}$, lire $0.2342717^{\circ}$. $20^{6}, 80^{\prime}$. Log sin. Au lieu de o.3200 $436 \mathrm{I}$, lire $0.3209436 \mathrm{r}^{4}$.

I. Les errata ci-dessus représentent toutes les erreurs signalées jusqu'ì présent dans les Tables pour le calcul des coordonjlées géodésiques par les formules de Benoit dans le cas de l'ellipsoïdc de réf'érence international (Bulletins. géodésiques $n^{20}{ }_{12}$, octobre-novembre-décembre i $\mathrm{g}^{2} \mathrm{l}$, et 16 , octobre-novembredécembre $19^{2} 7$ ), it dans les Tables à 8 dícimales des valeurs nalurelles des sinus, cosinus et tangentes dans le systéme décimal, etr., parues en février rọ?. 